\title{
LETTER OPEN \\ Longitudinal dynamics of antibody responses in recovered COVID-19 patients
}

Signal Transduction and Targeted Therapy (2021)6:137

\section{Dear Editor,}

The coronavirus disease 2019 (COVID-19) has resulted in more than 66.5 million cases globally in more than 191 countries with over 1,529,000 mortalities as of 6 December. Currently, the COVID19 continued to spread around the world, while the duration of antibody lasts and antibody level remained poorly understood, which are primary for safe and effective antiviral treatments and vaccines in the future. In this study, we comprehensively characterized the longitudinal changes of antibodies in recovered patients from 5-7 to 34-42 weeks after symptom onset.

We enrolled 24 COVID-19 recovered patients in this study, including 13 mild and 11 severe patients, respectively. We followed up the discharged patients and collected blood 1-5 times. To better analyze the dynamic responses over time, we divided the samples into 5 time points, including 5-7, 8-10, 11-13, 14-16, and 34-42 weeks after symptom onset, and each group all included $\mathrm{mild} / \mathrm{severe}$ recovered patients.

We firstly measured the IgG and IgM titers by ELISA. The IgG antibody was decreasing from 5-7 to 34-42 weeks (Fig. 1a), while there was no significant difference among different time points. Obviously, the IgG titers of the severe were higher than that of the mild, and statistically higher during 14-16 and 34-42 weeks (Fig. 1b). In addition, the lgG titers did not decline significantly over time in either mild or severe patients (Fig. 1b). These data suggested the IgG antibody against SARS-CoV-2 could be stable till 34-42 weeks in the recovered patients.

The IgM antibody against SARS-CoV-2 of the recovered subjects was all positive during 5-7 weeks, while during 8-11 weeks, $16.7 \%$ of the patients were negative for the IgM (Fig. 1c, d). And the number of negative samples for lgM increased gradually during the followed weeks (Fig. 1c, d). Compared with IgM during 5-7 weeks, there was no significant difference during 8-10 and 11-13 weeks, while showing significant reduction during 14-16 weeks (Fig. 1c, d).

Neutralizing antibodies are the critical correlates of protective immunity. As shown in Fig. 1e, $f$, with the extension of time, the $\mathrm{NT}_{50}$ titers were in a decreasing line from 5-7 to 11-13 and then maintained stable till 34-42 weeks. During 5-7 weeks, the average $\mathrm{NT}_{50}$ titers were at a high level of 1929 , while showed a significant drop to 644.6 during 8-10 weeks. Interestingly, the mean NT50 titers of the 3 followed time points maintained at a similar level that slightly lower than that during 8-10 weeks. Of note, the $\mathrm{NT}_{50}$ titers of severe patients were significantly higher than that of the mild from 5-7 to 34-42 weeks (Fig. 1f). We further compared the $\mathrm{NT}_{50}$ titers between patients who treated with corticosteroids or not, while the $\mathrm{NT}_{50}$ titers of the corticosteroids-patients were slightly higher than the non-corticosteroids-patients (Supplementary Fig. S1a). Then we found that the $\mathrm{NT}_{50}$ titers of the noncorticosteroids-patients were slightly higher than the corticosteroid patients from the limited number of mild patients (Supplementary Fig. S1b, c). It indicated that the humoral immune
; https://doi.org/10.1038/s41392-021-00559-7

responses were more robust in severe patients and corticosteroids may have blunted the neutralization response. ${ }^{1}$

Furthermore, regression analysis was performed on $\mathrm{NT}_{50}$ and the corresponding lgG titers (Fig. 1g). The correlation was weak of all the samples from 5 to 42 weeks. Then we analyzed the correlation at each time point separately. During 5-7 weeks, the $R^{2}$ was only 0.1535 , then increased to $0.5336,0.6344,0.5940$, and 0.6147 during $8-10,11-13,14-16$, and $34-42$ weeks, respectively, which was representing in an increasing line from 5-7 to 11-13 weeks and maintained stable till 34-42 weeks.

We also conducted a standard plaque reduction neutralization test (PRNT) to confirm the neutralization capacity and calculated the $50 \%$ neutralization titers $\left(\mathrm{PRNT}_{50}\right)$. Consistent with NT50 titers, PRNT50 titers of the severe was significantly higher than that of the mild during 5-7 and 14-16 weeks, and still maintained at a high level of 256.8 during 14-16 weeks (Fig. 1h). Moreover, the correlation was very strong between $\mathrm{NT}_{50}$ and PRNT 50 titers (Fig. 1i). A recent study ${ }^{2}$ has indicated that a 1:30 titer measured by PRNT $_{50}$ reduced the risk of getting reinfection by $50 \%$. According to the strong correlation between $\mathrm{NT}_{50}$ and $\mathrm{PRNT}_{50}$ titers in our study, and the geometric median and arithmetic mean of the ratios of $\mathrm{NT}_{50}$ and $\mathrm{PRNT}_{50}$ titers are 2.34 and 2.58 , respectively, so we suppose that the $\mathrm{NT}_{50}$ titer is at least 70.2 that the risk of reinfection can be reduced by $50 \%$.

Next, we calculated the neutralization potency index $\left(\mathrm{NT}_{50} / \mathrm{lgG}\right)$ to predict disease severity and survival as previously described. ${ }^{1}$ The neutralization potency index was similar from 5-7 to 14-16 weeks, while was significantly higher during 34-42 weeks (Fig. 1j). Besides a slightly higher in mild patients during 34-42 weeks, there was no difference between mild and severe patients from 5-7 to 14-16 weeks (Fig. 1k). Consistent with $\mathrm{NT}_{50}$ titers, the neutralization potency index of non-corticosteroidspatients was similar to corticosteroid patients in all enrolled patients (Fig. 1I), while was slightly higher in mild patients (Fig. $1 \mathrm{~m}$, Supplementary Fig. S1). Of note, the neutralization potency index showed an obvious increasing trend during 34-42 weeks.

Finally, we analyzed the positive proportion of $\lg G$, $\lg M$, and neutralizing antibodies in the recovered patients (Fig. 1n). The positive proportion of IgG and neutralizing antibodies were both $100 \%$ till 34-42 weeks after symptom onset. The proportion of patients with positive lgM antibody was $100 \%$ during 5-7 weeks, then gradually decreased to $50.0 \%$ during $34-42$ weeks. These results suggested that IgM antibody faded away in the recovered patients over time.

In our study, it is the first time to describe the correlation between $\operatorname{lgG}$ and neutralizing antibodies in detail. During 5-7 weeks the $R^{2}$ was only 0.1535 , which may account for the wave of short-lived, low-affinity antibodies generated by plasmablasts during acute SARS-CoV-2 infection, which then gradually degraded and the germinal center responses that generated highaffinity antibodies can maintain for months to many years. ${ }^{3}$ We 

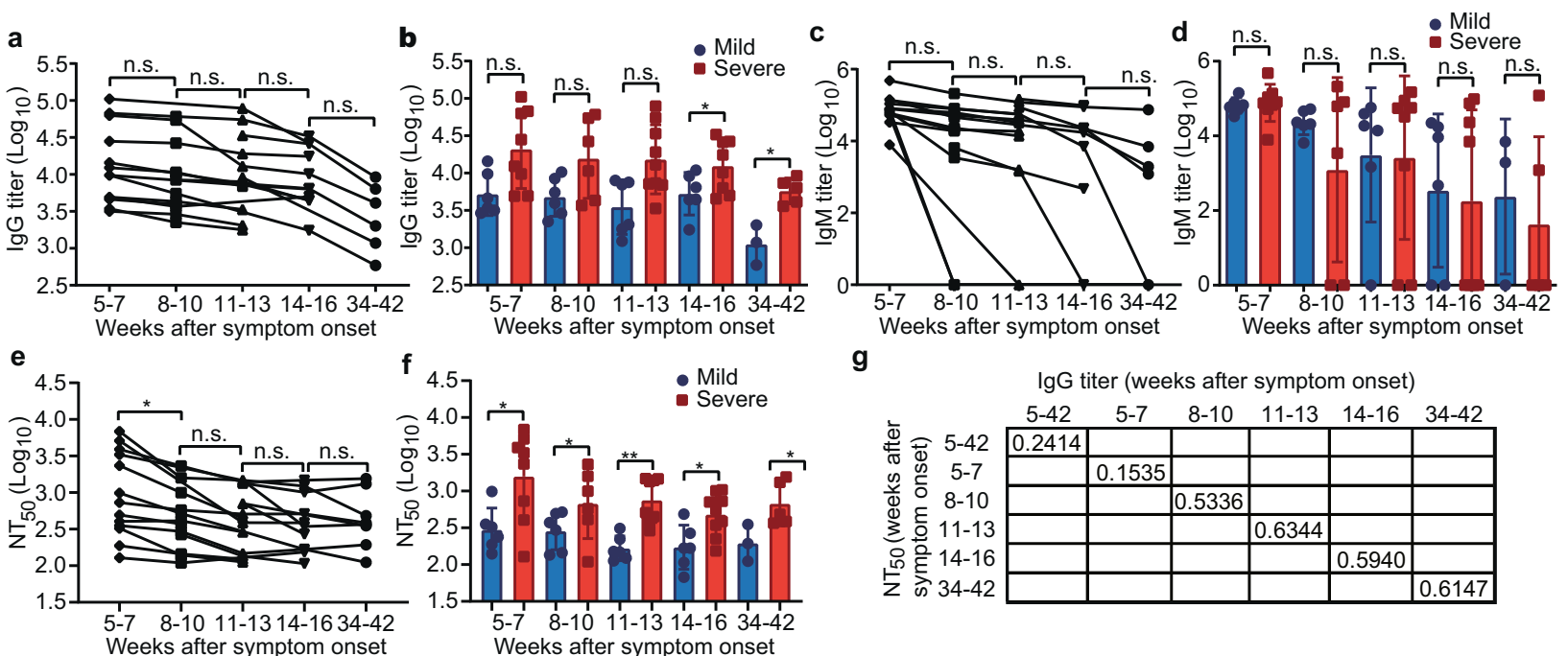

g

\begin{tabular}{|c|c|c|c|c|c|c|}
\hline \multirow{3}{*}{$\stackrel{\bar{\Phi}}{ \pm}=5-42$} & \multicolumn{6}{|c|}{ IgG titer (weeks atter symptom onset) } \\
\hline & $5-42$ & $5-7$ & 8-10 & $11-13$ & $14-16$ & $34-42$ \\
\hline & 0.2414 & & & & & \\
\hline \multirow{5}{*}{ 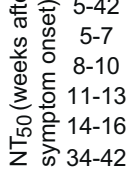 } & & 0.1535 & & & & \\
\hline & & & 0.5336 & & & \\
\hline & & & & 0.6344 & & \\
\hline & & & & & 0.5940 & \\
\hline & & & & & & 0.6147 \\
\hline
\end{tabular}
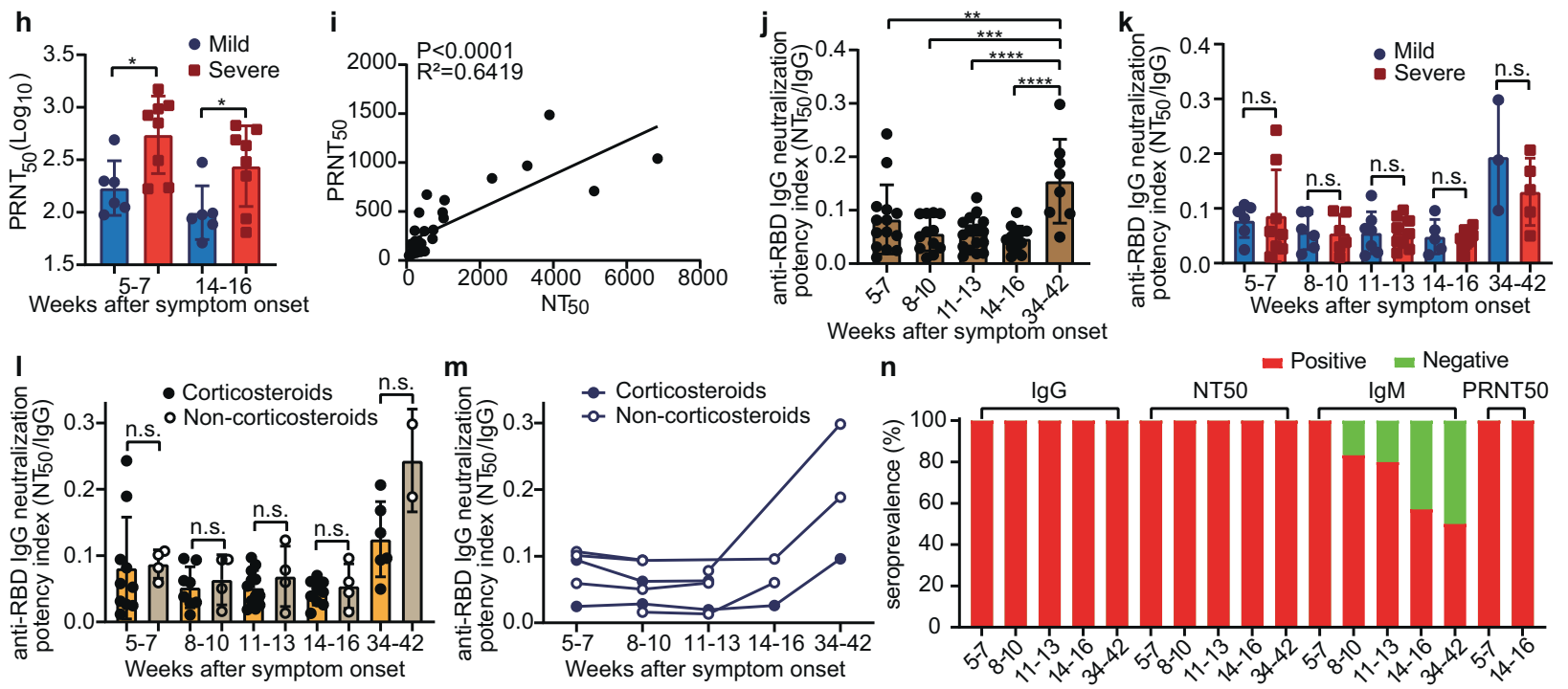

Weeks after symptom onset

Fig. 1 Antibody responses against SARS-CoV-2 spike protein in COVID-19 recovered individuals. IgG (a), lgM (c), and NT 50 titers (e) against SARSCoV-2 S-RBD or S protein of the recovered patients from 5-7 to 34-42 weeks $(n=17)$. Comparison of the lgG (b), IgM (d), and NT 50 titers $(\mathbf{f})$ between mild and severe patients from 5-7 to 34-42 weeks $(n=24)$. g Correlation analysis of $\mathrm{NT}_{50}$ titers and lgG titers of the recovered patients during 5-42, $5-7,8-10,11-13,14-16$, and 34-42 weeks. h Comparison of the PRNT 50 titers between mild and severe patients during 5-7 $(n=14)$ and $14-16(n=$ 14) weeks. $i$ Correlation analysis of $\mathrm{NT}_{50}$ titers and $\mathrm{PRNT}_{50}$ titers of the recovered patients during 5-16 weeks $(n=28)$. Anti-RBD lgG neutralization potency index ( $\mathrm{NT}_{50} / \mathrm{lgG}$ ) was calculated (j) and compared between mild and severe patients (k) from 5-7 to 34-42 weeks $(n=24)$. I Comparison of the anti-RBD lgG neutralization potency index ( $\mathrm{NT}_{50} / \mathrm{lgG}$ ) between the patients who treated with corticosteroids or not from 5-7 to 34-42 weeks $(n=24)$. $\mathbf{m}$ The longitudinal dynamics of the anti-RBD lgG neutralization potency index $\left(\mathrm{NT}_{50} / \mathrm{lgG}\right)$ in mild patients who treated with corticosteroids or not $(n=7)$. $\mathbf{n}$ Seroprevalence of the lgG, IgM, NT 50 , and PRNT 50 titers in patients from 5-7 to 34-42 weeks after symptom onset (5-7, $n=14 ; 8-10$, $n=12 ; 11-13, n=17 ; 14-16, n=10 ; 34-42, n=8)$. n.s., not significant; ${ }^{*} P<0.05 ;{ }^{* *} P<0.01 ;{ }^{* * *} P<0.001 ;{ }^{* * *} P<0.0001$

find that the strong correlation between $\lg G$ and neutralizing antibodies was appeared during 11-13 weeks, while the plasmablasts are no longer present in recovered individuals at $\sim 1$ month after symptom onset, ${ }^{4}$ that on one hand it probably because of short-lived, low-affinity antibodies degraded later than plasmablasts disappeared, on the other hand, it possibly account for the time distance between plasmablasts disappeared and the germinal center generated high-affinity antibodies.

The humoral immune responses play a critical role in the clearance of cytopathic viruses and prevention of viral reinfection. Since the outbreak of COVID-19, scientists are committed to exploring the nature and durability of the humoral immune response. Recently, Ripperger et al. ${ }^{5}$ found that neutralizing antibodies were durable at least 2-3 months and Wajnberg et al. ${ }^{2}$ found that more than $90 \%$ were detectable for at least 5-7 months after SARS-CoV-2 infection in the mild-to-moderate COVID-19. While in our cohort, the positive proportion of neutralizing antibodies was $100 \%$, and the $\mathrm{NT}_{50}$ titers in all patients were $>110$ till 34-42 weeks after symptom onset. Our results indicated that the high-affinity and efficiency-neutralizing antibodies could last for at least 10 months and supported protective immunity in the recovered patients, and provided insight on the rational development of neutralizing antibodies based on preventive and therapeutic strategies against COVID-19 in the future.

\section{ACKNOWLEDGEMENTS}

This work was supported by the National Key Research and Development Project of China (2020YFC0842200, 2020YFA0707801), the Beijing Natural Science Foundation (No. 7202196), Beijing Nova Program (No. xx2018052), and Science and Technology Development Plan Project of Jilin Province (20200901007SF). Cheng-Feng Qin was supported by the National Science Fund for Distinguished Young Scholar (No. 81925025), and the Innovation Fund for Medical Sciences (No. 2019RU040) from the Chinese Academy of Medical Sciences (CAMS). 


\section{AUTHOR CONTRIBUTIONS}

C.F.Q., C.Q.B., M.L.C., H.Y.L., F.L., and G.Q.W. contributed to the study design, data analysis, and interpretation. C.F.Q., M.L.C., and H.Z. contributed to writing and editing the manuscript. H.Y.L. and J.Z. contributed to collecting clinical specimens. M.L.C., H. Z., X.F.L., and C.Z. contributed to performing all the experiments.

\section{ADDITIONAL INFORMATION}

Supplementary information The online version contains supplementary material available at https://doi.org/10.1038/s41392-021-00559-7.

Competing interests: The authors declare no competing interests.

Meng-Li Cheng ${ }^{1,2}$, Hui-Ying Liu ${ }^{3}$, Hui Zhao ${ }^{2}$, Guo-Qing Wang (D) Chao Zhou ${ }^{2}$, Jing Zheng ${ }^{3}$, Xiao-Feng $\mathrm{Li}^{2}$, Fan $\mathrm{Li}^{1}$

Chang-Qing Bai ${ }^{3}$ and Cheng-Feng Qin (iD ${ }^{2}$

${ }^{1}$ College of Basic Medical Science, Jilin University, Changchun, China;

${ }^{2}$ Department of Virology, State Key Laboratory of Pathogen and

Biosecurity, Beijing Institute of Microbiology and Epidemiology, Academy of Military Medical Sciences, Beijing, China and

${ }^{3}$ Department of Respiratory and Critical Care Diseases, the Fifth

Medical Center, Chinese PLA General Hospital, Beijing, China

These authors contributed equally: Meng-Li Cheng, Hui-Ying Liu, Hui

Zhao, Guo-Qing Wang.

Correspondence: Fan Li (lifan@jlu.edu.cn) or Chang-

Qing Bai (baica307@163.com) or Cheng-Feng Qin (qincf@bmi.ac.cn)

\section{REFERENCES}

1. Garcia-Beltran, W. F. et al. COVID-19-neutralizing antibodies predict disease severity and survival. Cell 184, 476-488 e411 (2021)

2. Wajnberg, A. et al. Robust neutralizing antibodies to SARS-CoV-2 infection persist for months. Science 370, 1227-1230 (2020).

3. Slifka, M. K. \& Ahmed, R. Long-term antibody production is sustained by antibodysecreting cells in the bone marrow following acute viral infection. Ann. N. Y. Acad. Sci. 797, 166-176 (1996).

4. Mathew, D. et al. Deep immune profiling of COVID-19 patients reveals distinct immunotypes with therapeutic implications. Science 369, eabc8511 (2020).

5. Ripperger, T. J. et al. Orthogonal SARS-CoV-2 serological assays enable surveillance of low-prevalence communities and reveal durable humoral. Immun. Immun. $\mathbf{5 3}$ 925-933 (2020).

(1)

Open Access This article is licensed under a Creative Commons Attribution 4.0 International License, which permits use, sharing, adaptation, distribution and reproduction in any medium or format, as long as you give appropriate credit to the original author(s) and the source, provide a link to the Creative Commons license, and indicate if changes were made. The images or other third party material in this article are included in the article's Creative Commons license, unless indicated otherwise in a credit line to the material. If material is not included in the article's Creative Commons license and your intended use is not permitted by statutory regulation or exceeds the permitted use, you will need to obtain permission directly from the copyright holder. To view a copy of this license, visit http://creativecommons. org/licenses/by/4.0/.

(c) The Author(s) 2021 